OPEN ACCESS

Edited and reviewed by

Christoph T. Berger,

University of Basel, Switzerland

*Correspondence:

Taisheng Li

litsh@263.net

${ }^{\dagger}$ These authors have contributed equally to this work

Specialty section: This article was submitted to

Vaccines and Molecular Therapeutics,

a section of the journal

Frontiers in Immunology

Received: 23 February 2021 Accepted: 04 March 2021 Published: 22 March 2021

Citation:

Cao W, Liu X, Hong K, Ma Z

Zhang $Y$, Lin L, Han $Y$, Xiong $Y$,

Liu Z, Ruan L and Li T (2021) Corrigendum: High-Dose Intravenous Immunoglobulin in Severe Coronavirus

Disease 2019: A Multicenter

Retrospective Study in China.

Front. Immunol. 12:671443.

doi: 10.3389/fimmu.2021.671443

\section{Corrigendum: High-Dose Intravenous Immunoglobulin in Severe Coronavirus Disease 2019: A Multicenter Retrospective Study in China}

\author{
Wei Cao ${ }^{1 \dagger}$, Xiaosheng Liu ${ }^{1,2,3 \dagger}$, Ke Hong ${ }^{4}$, Zhiyong Ma ${ }^{5}$, Yuelun Zhang ${ }^{6}$, Ling Lin ${ }^{1}$, \\ Yang Han ${ }^{1}$, Yong Xiong ${ }^{5}$, Zhengyin Liu ${ }^{1}$, Lianguo Ruan ${ }^{4}$ and Taisheng $\mathrm{Li}^{1,2,3^{*}}$ \\ ${ }^{1}$ Department of Infectious Diseases, Peking Union Medical College Hospital, Peking Union Medical College and Chinese \\ Academy of Medical Sciences, Beijing, China, ${ }^{2}$ Department of Basic Medical Sciences, School of Medicine, Tsinghua \\ University, Beijing, China, ${ }^{3}$ Tsinghua-Peking Center for Life Sciences, Beijing, China, ${ }^{4}$ Department of Infectious Diseases, \\ JinYin-tan Hospital, Wuhan, China, ${ }^{5}$ Department of Infectious Diseases, Zhongnan Hospital of Wuhan University, Wuhan, \\ China, ${ }^{6}$ Medial Research Center, Peking Union Medical College Hospital, Peking Union Medical College and Chinese \\ Academy of Medical Sciences, Beijing, China
}

Keywords: COVID-19, high-dose intravenous immunoglobulin, immunomodulation, 28-day mortality, inflammatory markers

\section{A Corrigendum on}

High-Dose Intravenous Immunoglobulin in Severe Coronavirus Disease 2019: A Multicenter Retrospective Study in China

By Cao W, Liu XS, Hong K, Ma ZY, Zhang YL, Lin L, et al. (2021). Front. Immunol. doi: $10.3389 /$ fimmu.2021.627844

\section{ERROR IN TABLE}

In the original article, there was a mistake in Table $\mathbf{1}$ as published. The author identified the calculating errors in the percentage of comorbidity, symptoms, and treatment. The corrected Table 1 appears below.

The authors apologize for this error and state that this does not change the scientific conclusions of the article in any way. The original article has been updated.

Copyright $\odot 2021$ Cao, Liu, Hong, Ma, Zhang, Lin, Han, Xiong, Liu, Ruan and Li. This is an open-access article distributed under the terms of the Creative Commons Attribution License (CC BY). The use, distribution or reproduction in other forums is permitted, provided the original author(s) and the copyright owner(s) are credited and that the original publication in this journal is cited, in accordance with accepted academic practice. No use, distribution or reproduction is permitted which does not comply with these terms. 
TABLE 1 | Clinical and relevant baseline characteristics of patients.

\begin{tabular}{|c|c|c|c|c|}
\hline Characteristic & Total $(n=115)$ & IVIg group $(n=26)$ & Control group $(n=89)$ & SD (\%) \\
\hline \multicolumn{5}{|l|}{ Demographics } \\
\hline Age, median (IQR) & $59(47-69)$ & $58(42-65)$ & $59(48-70)$ & 16 \\
\hline Male sex, No. (\%) & $76(66)$ & $19(73)$ & $57(64)$ & 18 \\
\hline \multicolumn{5}{|l|}{ Any comorbidity, No. (\%) } \\
\hline Hypertension & $52(45)$ & $10(38)$ & $42(47)$ & 9 \\
\hline Diabetes & $24(21)$ & $2(8)$ & $22(25)$ & 39 \\
\hline Chronic cardiac disease & $18(16)$ & $5(19)$ & $13(15)$ & 11 \\
\hline Chronic respiratory disease & $11(10)$ & $2(8)$ & $9(10)$ & 4 \\
\hline \multicolumn{5}{|l|}{ Any symptoms, No. (\%) } \\
\hline Fever & $111(97)$ & $22(85)$ & $89(100)$ & 31 \\
\hline Cough & $97(84)$ & $22(85)$ & $75(84)$ & 8 \\
\hline Dyspnea & $96(83)$ & $22(85)$ & $74(83)$ & 10 \\
\hline Fatigue or myalgia & $52(45)$ & $9(35)$ & $43(48)$ & 19 \\
\hline Diarrhea & $30(26)$ & $4(15)$ & $26(29)$ & 24 \\
\hline \multicolumn{5}{|l|}{ Vital signs } \\
\hline Systolic BP, mm Hg & $132 \pm 19$ & $131 \pm 14$ & $133 \pm 20$ & 5 \\
\hline $\mathrm{HR}, / \mathrm{min}$ & $88(80-102)$ & $85(81-100)$ & $89(80-105)$ & 9 \\
\hline $\mathrm{RR}$, breaths/min & $22(20-25)$ & 22 (20-23) & 22 (20-25) & 2 \\
\hline $\mathrm{SPO}_{2}, \%$ & $90(87-94)$ & 91 (89-94) & 89 (86-92) & 6 \\
\hline \multicolumn{5}{|l|}{ Complete blood count } \\
\hline WBCs, $\times 10^{9} / \mathrm{L}$ & $6.59(4.38-9.64)$ & $6.25(4.15-10.28)$ & $6.60(4.42-9.56)$ & 4 \\
\hline $\mathrm{LYM}, \times 10^{9} / \mathrm{L}$ & $0.76(0.57-1.02)$ & $0.90(0.62-1.05)$ & $0.73(0.56-0.91)$ & 19 \\
\hline $\mathrm{PLT}, \times 10^{9} / \mathrm{L}$ & $191(148-269)$ & $172(144-279)$ & $199(150-270)$ & 18 \\
\hline \multicolumn{5}{|l|}{ Inflammatory biomarkers } \\
\hline IL-6, pg/mL & $13.0(8.0-31.5)$ & 15.5 (10.5-34.0) & $11.0(7.2-32.2)$ & 15 \\
\hline $\mathrm{IL}-8, \mathrm{pg} / \mathrm{mL}$ & $15.2(6.0-27.3)$ & $16.2(5.8-23.8)$ & $15.2(5.9-28.4)$ & 3 \\
\hline $\mathrm{IL}-10, \mathrm{pg} / \mathrm{mL}$ & $5.2(5.0-10.3)$ & $5.0(5.0-8.5)$ & $5.5(5.0-11.2)$ & 2 \\
\hline $\mathrm{hsCRP}, \mathrm{mg} / \mathrm{mL}$ & $48(17-94)$ & $28(7-91)$ & $45(17-101)$ & 14 \\
\hline Ferritin, ng/mL & 807.0 (473.4-1383.3) & 774.1 (444.2-1525.4) & $838.8(501.5-1351.1)$ & 18 \\
\hline $\mathrm{ESR}, \mathrm{mm} / \mathrm{h}$ & $50(25-65)$ & 35 (19-67) & $50(27-65)$ & 14 \\
\hline \multicolumn{5}{|l|}{ Immunoglobulins } \\
\hline $\lg A, g / L$ & $2.2(1.8-3.0)$ & $2.7(1.9-4.6)$ & $2.2(1.6-3.0)$ & 2 \\
\hline $\lg G, g / L$ & $11.1(9.0-13.1)$ & $11.8(8.5-15.7)$ & $11.1(9.3-13.1)$ & 2 \\
\hline $\operatorname{lgM} \mathrm{g} / \mathrm{L}$ & $1.0(0.7-1.2)$ & $1.0(0.9-1.1)$ & $0.9(0.7-1.3)$ & 3 \\
\hline Seven-category scales at admission & $4.0 \pm 0.67$ & $3.85 \pm 0.67$ & $4.06 \pm 0.66$ & 26 \\
\hline 3, No. (\%) & $20(17)$ & $7(27)$ & $13(15)$ & 24 \\
\hline 4 , No. (\%) & 79 (69) & $17(65)$ & $62(70)$ & 7 \\
\hline 5, No. (\%) & $11(10)$ & $1(4)$ & $10(11)$ & 25 \\
\hline 6 , No. (\%) & $5(4)$ & $1(4)$ & $4(4)$ & 3 \\
\hline Murray lung injury scores at admission & $3.93 \pm 0.22$ & $3.90 \pm 0.28$ & $3.94 \pm 0.20$ & 11 \\
\hline Time to admission after onset, median (IQR) & $10(7-12)$ & $10(7-12)$ & $10(8-12)$ & 5 \\
\hline Earlier (< 7 days), No. (\%) & $29(25)$ & $7(27)$ & $22(25)$ & 12 \\
\hline Later (between 7-14 days), No. (\%) & $86(75)$ & $19(73)$ & $67(75)$ & 12 \\
\hline \multicolumn{5}{|l|}{ Treatment during hospitalization, No. (\%) } \\
\hline Antiviral treatment & $100(87)$ & $22(85)$ & $78(88)$ & 7 \\
\hline Arbidol & $75(65)$ & $15(58)$ & $60(67)$ & 16 \\
\hline $\mathrm{IFN}-\alpha$ & $34(30)$ & $14(54)$ & $20(22)$ & 53 \\
\hline $\mathrm{LPV} / \mathrm{r}$ & $31(27)$ & $9(35)$ & $22(25)$ & 17 \\
\hline RBV & $12(10)$ & $3(12)$ & $9(10)$ & 4 \\
\hline OSV & $33(29)$ & $8(31)$ & $25(28)$ & 5 \\
\hline Antibiotic treatment & $100(87)$ & $25(96)$ & $75(84)$ & 37 \\
\hline Moxifloxacin & $73(63)$ & $15(58)$ & $58(65)$ & 12 \\
\hline Cefoperazone and tazobactam & $47(41)$ & $13(50)$ & $34(38)$ & 19 \\
\hline Antifungal treatment & $11(10)$ & $5(19)$ & $6(7)$ & 29 \\
\hline TCM & $58(50)$ & $15(58)$ & $43(48)$ & 15 \\
\hline LMWH & $19(16)$ & $4(15)$ & $15(17)$ & 3 \\
\hline Glucocorticoids & $71(62)$ & $18(69)$ & $53(60)$ & 17 \\
\hline IVIg therapy & $26(23)$ & $26(100)$ & $0(0)$ & - \\
\hline Initiation time, days, median (IQR) & - & $2(1-4)$ & - & - \\
\hline Course, days, median (IQR) & - & $5(5-9)$ & - & - \\
\hline Amount, g, median (IQR) & - & 122.5 (95.0-213.8) & - & \\
\hline
\end{tabular}

$S D$, standard deviation; Tmax, maximal temperature; $B P$, blood pressure; HR, heart rate; $R R$, respiratory rate; $S P \mathrm{O}_{2}$, pulse oximeter $\mathrm{O} 2$ saturation; WBC, white cell counts; $L Y M$, lymphocyte counts; PLT, platelet counts; IL, interleukin; CRP, C-reactive protein; ESR, erythrocyte sedimentation rate; IFN- $\alpha$, Interferon-alfa; LPV/r, Iopinavir/ritonavir; RBV, ribavirin; OSV, oseltamivir; TCM, traditional Chinese medicine; LMWH, low molecular weight heparin. 